\title{
How generalisable are empirical models to estimate high resolution spatial indicators of crop performance at the regional scale?
}

\author{
Lyle, G., Arbon, K., Clarke, K., Kilpatrick, A., Summers, D. and Ostendorf, B. \\ Landscape Science, Earth and Environmental Science, The University of Adelaide \\ Email: gregory.lyle@adelaide.edu.au
}

\begin{abstract}
Climate change mitigation and adaptation responses in agricultural areas will be a balance between strategies such as the allocation of land to different land uses and changes to current land management. Identification of the spatial variability of production is fundamental to both strategies if efficient area-based decisions are to be made which minimise on-farm economic losses and food security and sustainability issues. Also critical to these strategies is that the information created must be at a resolution high enough to enable on-farm decisions and at extents large enough to guide targeted governmental policy.

This paper aims to address these needs by developing a method for mapping spatial variation in yield at a high resolution across the cropping areas in South Australia.

We developed regression models between satellite-derived Normalised Difference Vegetation Index (NDVI) and yield measured through precision agriculture technologies across three years and compared their predictions with regional yield statistics.

Observations and predictions of district average grain yields ranged from 0.5 to $3.3 \mathrm{t} / \mathrm{ha}$ during the study period. The best model efficiency criterion (Nash-Sutcliffe) was $65 \%$ in 2005. Our results demonstrate that empirical relationships between high resolution spatial datasets are spatially representative. That is, a model based on the yield-NDVI relationship developed in a very small area can be used to accurately predict regional yield.
\end{abstract}

Keywords: Yield mapping, Landsat, prediction, evaluation 


\section{INTRODUCTION}

Of all economic sectors, agriculture will be among the most affected by anthropogenic climate change. While the specific impact of climate change on yields will vary geographically, information is needed on the spatial distribution of yield with high spatial resolution and over extensive areas. Both magnitude and the variability of yield are important factors that need consideration for management. Decision making will involve climate change mitigation and adaptation strategies implemented both independently and cooperatively between farmers and governments which rely on the allocation of land to different land uses and changes to current land management.

Identification of the spatial variability in production is fundamental for farm level decision making such as area allocation for different land uses. Efficient area-based decisions need to maximise on-farm income considering sustainability issues as well as potential income from government incentives. Precision agriculture is an integral component of modern farm management that has become an important source of information to locate low productivity areas in need for management alternatives. However, management at broader scale usually only uses aggregated data that disregards this kind of variability.

Past research has shown that yield data correspond well with satellite imagery and can be used to map spatial pattern of yield (Lyle and Ostendorf, 2011) and that LANDSAT imagery at different dates shows significant relationships with yield (Lyle et al., 2013). Previous work used detailed data from a limited geographic extent and applicability to other regions is untested.

In this paper, we present an evaluation if yield models based on NDVI can be used to predict broad-scale yield statistics. We collated yield data from 98 paddocks (total area of $13.5 \mathrm{~km}^{2}$ ) and extrapolated to an area around $40,000 \mathrm{~km}^{2}$.

\section{STUDY AREA AND STUDY PERIOD}

The study focuses on the extensive South Australian cropping region, an area of approximately eight million hectares. The climate in this area is Mediterranean, with hot dry summers from December to February and mild wet winters from July to August. Rainfall falls predominantly in winter and spring, and ranges from $250 \mathrm{~mm}$ in the north to $600 \mathrm{~mm}$ in the south. Agriculture in the region is dominated by cereals crops, but is broken up by rotating legumes, pasture and fallow.

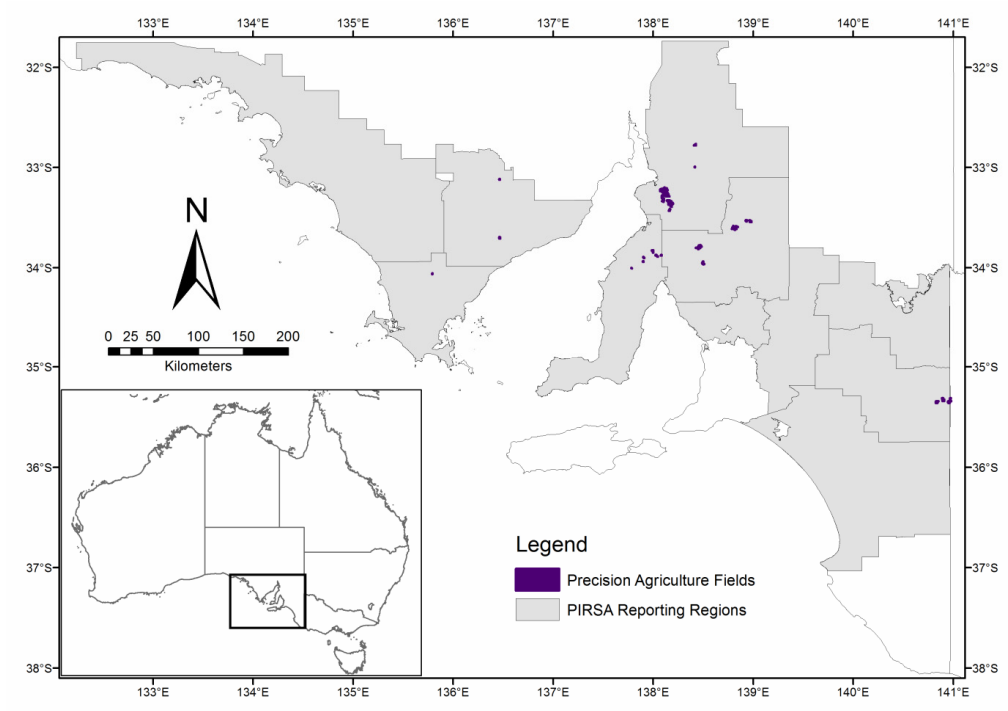

Figure 1. Location and distribution of precision agriculture fields and Primary Industries and Resources SA (PIRSA) reporting regions.

To investigate the climatic sensitivity of our models it was necessary for the study to incorporate years with a range of climatic conditions, ranging from poor (for cereal crop production), through medium to good. The period 2004 to 2006 was identified as suitable. Average cereal crop yields were recorded in 2004, in 2005 higher yields were achieved, while 2006 was a very dry year and consequently lower yields were produced. 
Yield data access was provided by growers belonging to the Southern Precision Agriculture Association (SPAA). This organisation specifically deals growers interested in progressing precision agriculture technology, adoption and management in South Australia. Figure 1 shows the spatial distribution of fields used in the analysis over the years 2004, 2005 and 2006 highlighting the lack of yield data obtained in some areas.

\section{SATELLITE IMAGERY}

\subsection{Creation of image products}

Two basic image products were produced; a crop type classification (wheat or barley) and a composite map of Normalised Difference Vegetation Index (NDVI). Together, the crop type classification and the NDVI images allowed generation of the crop specific empirical NDVI/yield relationships.

\subsection{Image data}

Landsat 5 TM and Landsat 7 ETM+ data was used in this study, and obtained from the USGS Landsat archive at level 1T (precision and terrain corrected). Both sensors have resolutions of 30 metres, $185 \times 185$ $\mathrm{km}$ extents and a revisit frequency of 16 days. It bears mentioning that the Landsat 7 sensor developed a fault in July 2003 with the Scan Line Corrector (SLC). The SLC module compensated for the forward motion of the satellite, and the failure of this module has resulted in bands of 'no data' at the edges of the scenes. However, Landsat 7 imagery is still radiometrically sound.

\subsection{Image acquisition}

Image date selection was based on published research into the association between final grain yield and remotely sensed estimates of biomass measured. In Australia, prediction of wheat yield from NDVI using the Landsat sensor has been found to be most accurate with imagery acquired between August and October, with imagery acquired in September being optimal (Lyle et al., 2013; Shut et al., 2009). For this period, all Landsat images in the USGS archive from 2004 to 2006 were examined, and images with no cloud cover over cropping areas were selected for analysis. It is important to note that the imagery acquired must be cloud free as cloud moisture effects will influence the NDVI signal. Ten cloud free images were selected for each year which covered the majority of the South Australian cropping region. These images were mosaiced together to form three annual images. Prior to classification and NDVI calculation all images were corrected to top-of-atmosphere reflectance using the methods and variables detailed in Chandler et al., 2009.

\subsection{Crop type classification}

Supervised classification was used to map three crop types (wheat, barley or other) across the study area from training signatures taken from fields within one farm in the Crystal Brook area. These fields were chosen for signature collection because they were spatially extensive, providing a large number of training pixels and a detailed recording of crop type information across the study period. Signatures were extracted based on the field boundaries, minus a $30 \mathrm{~m}$ buffer to ensure mixed boundary pixels were excluded. Classification was performed on each of the three annual images in ERDAS Imagine, and used the maximum likelihood decision rule. A priori probabilities were used to help determine the appropriate mix of crops on ground based on the percentage breakdown of wheat and barley crops across the SA cropping region over a ten year period provided by Primary Industries and Resources SA (PIRSA) agricultural statistics (PIRSA, 2009). Usually in agriculture, only one crop is sown in each field. However, the above classification was per-pixel, and therefore mapped a speckled pattern of both wheat and barley in most fields. The South Australian cadastral database was used to determine field extents and these fields were assigned to either wheat or barley crop type based on which crop type had the greater proportion of classified pixels within that field.

\subsection{Accuracy assessment of crop type}

Accuracy of classification was tested against crop histories provided by SPAA for fields throughout the study area. Producer's accuracy - the probability of a reference pixel being correctly classified indicating how well an area can be classified and User's accuracy - is the probability that a pixel classified represents that category on ground were calculated. 
This represented 68 fields for 2004, 117 fields for 2005 and 106 fields for 2006. Wheat and barley crop types represented around $80 \%$ of the fields used in the accuracy assessment.

\subsection{Calculation of the NDVI}

NDVI was calculated for each year using the following index formula:

$$
\text { NDVI }=((\text { Band4-Band3 }) /(\text { Band4 +Band3 }))
$$

For each year's spatial distribution of crop type, NDVI values were extracted based on the locations of the wheat and barley crops identified by crop type classification. This index provides an estimation of wheat and barley plant biomass. Here, higher NDVI values indicate higher plant growth and in turn higher final grain yield.

\section{CREATION OF YIELD MAPPED GRAIN YIELD ESTIMATES}

\subsection{Post processing of yield mapped datasets}

Yield mapping data was run through the University of Adelaide post processing error removal software (Lyle, 2010). The software removed errors associated with harvester dynamics, the collection of measurements to calculate grain yield, the global positioning system and the driving of the combine operator. The implementation of such routines on these datasets produces yield distributions with a more normal distribution than that observed in the raw datasets (Blackmore, 2003).

\subsection{Interpolation of grain yield from yield datasets}

To establish yield estimates at the same spatial resolution as the Landsat imagery, all yield mapping datasets were interpolated using the Australian Centre for Precision Agriculture (ACPA) VESPER kriging software (Minasny et al., 2005). Wheat and barley grain yield surfaces were created at a grid resolution of $30 \mathrm{~m}$ using the ACPA yield mapping creation protocol (Taylor et al., 2007). The interpolation process produced a spatial structure for grain yield estimates across missing areas due to error removal and acted as a geographic basis for spatial comparison of wheat yield values and satellite based measurements.

\subsection{Relationships between grain yield and NDVI}

Fine-scale annual NDVI to yield relationships were developed for barley and wheat for each of the three study years. The $30 \mathrm{~m}$ pixels in the interpolated field yield maps were randomly divided into training and validation sets based on a 50:50 ratio and then matched with the corresponding Landsat NDVI value for that location and year. Power regression relationships were then fitted to the data for each crop type and year.

\subsection{Evaluation of yield predictions}

Two levels of validation were performed on the NDVI yield predictions.

Firstly, a fine scale evaluation of the yield-NDVI relationships was performed by comparing yield predicted from NDVI against the withheld validation pixels from the interpolated yield maps. The accuracy of the annual yield-NDVI relationships was evaluated against the validation dataset with two efficiency criteria, root mean square error (RMSE), and the Nash-Sutcliffe efficiency criteria $(E)$. The calculation of RMSE allows for the comparison of model prediction accuracy across models since it normalises the prediction error across varying yield distributions used in the validation process. $E$ measures how good a predictor the model is compared to using the average yield value for prediction (Nash and Sutcliffe, 1970). Values of $E$ range from 1 (indicating a perfect model fit) to $-\infty$, with values lower than zero indicating that the mean of the observed set would have been a better predictor than the model.

Secondly, a broad scale evaluation compared yield-NDVI predictions aggregated to PIRSA regional scale boundaries (Figure 1) and PIRSA surveyed regional yield estimates (PIRSA, 2009). Evaluation will identify whether the yield-NDVI relationships at the regional scale hold across areas where the yield data for the model was not available. The total annually predicted yield for each of the 11 regions was compared to the reported yield for that region. This process was performed for each study year and $E$ was calculated to determine model performance. 


\section{RESULTS}

\subsection{Crop type classification accuracy}

The overall accuracy of the crop type classification for 2004, 2005 and 2006 was poor. The producer's accuracy highlights the probability that the reference paddock is being correctly classified. Across all years the producer's accuracy was low - less than $50 \%$ across all crop types (Table 1 ). The probability that a paddock classified on the map actually represent that category on the ground (user's accuracy) provides slightly better results, especially in 2005 where around $60 \%$ of the wheat and barley paddocks were correctly located.

Table 1. Producer's and User's accuracies (\%) of the supervised crop type classifications for 2004, 2005 and 2006.

\begin{tabular}{|c|c|c|c|c|c|c|}
\hline \multirow[b]{2}{*}{ Crop } & \multicolumn{2}{|c|}{2004} & \multicolumn{2}{|c|}{2005} & \multicolumn{2}{|c|}{2006} \\
\hline & $\begin{array}{l}\text { Producer's } \\
\text { accuracy }(\%)\end{array}$ & $\begin{array}{l}\text { User's accuracy } \\
(\%)\end{array}$ & $\begin{array}{l}\text { Producer's } \\
\text { accuracy }(\%)\end{array}$ & $\begin{array}{l}\text { User's accuracy } \\
(\%)\end{array}$ & $\begin{array}{l}\text { Producer's } \\
\text { accuracy }(\%)\end{array}$ & $\begin{array}{l}\text { User's } \\
\text { accuracy }(\%)\end{array}$ \\
\hline Wheat & 45 & 52 & 44 & 66 & 37 & 52 \\
\hline Barley & 18 & 30 & 43 & 57 & 47 & 57 \\
\hline Other & 44 & 28 & 36 & 11 & 14 & 7 \\
\hline
\end{tabular}

\subsection{Fine-scale annual yield prediction} evaluation

The number of paddocks and interpolated $30 \mathrm{~m}$ yield measurements used for model training and validation for each crop type and year are presented in Table 2. In 2004, the weather was moderately conducive to cereal crop growth, and the relationship between yield and NDVI was weak for wheat and strong for barley (Table 2). In 2005 , growing conditions for cereal crops were better and the yield-NDVI relationship was moderate for both wheat and barley with an $\mathrm{R}^{2}$ of 0.53 and 0.49 respectively.

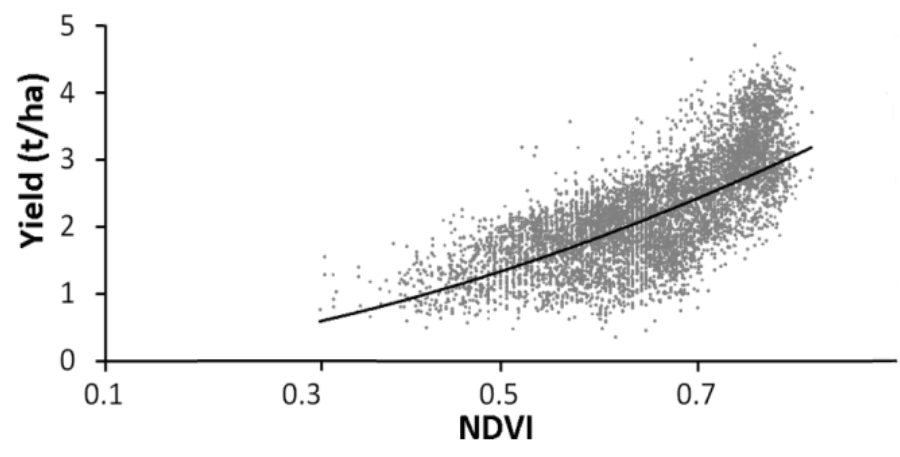

Figure 2. Local wheat yield predicted from NDVI for 2005 Figure 2 shows the relationships for wheat that indicates a strong relationship with a high variability around the regression line. In 2006, which was a dry year, the yield-NDVI relationship was strong for wheat but weak for barley. Absolute prediction errors were fairly similar for both crop types across all years, ranging from $0.52 \mathrm{t} / \mathrm{ha}$ RMSE for wheat in 2004 to $0.78 \mathrm{t} / \mathrm{ha}$ RMSE for barley in 2005 .

Table 2. Number of paddocks used and $30 \mathrm{~m}$ observations, derived power models and evaluation statistics for wheat and barley yield predictions for 2004, 2005 and 2006.

\begin{tabular}{|c|c|c|c|c|c|c|c|}
\hline Year & Crop & $\begin{array}{l}\text { Paddocks } \\
\text { used }\end{array}$ & $\begin{array}{l}\text { Number of } \\
30 \mathrm{~m} \\
\text { observations }\end{array}$ & $\begin{array}{l}\text { Power } \\
\text { models }\end{array}$ & $\mathrm{R}^{2}$ & $\begin{array}{l}\text { RMSE } \\
(\mathrm{t} / \mathrm{ha})\end{array}$ & $\begin{array}{l}\text { Efficiency } \\
\text { criteria }(E)\end{array}$ \\
\hline 2004 & Wheat & 48 & 6,800 & $y=2.21 x^{0.32}$ & 0.11 & 0.54 & 0.11 \\
\hline 2004 & Barley & 17 & 3,600 & $y=4.00 x^{0.74}$ & 0.48 & 0.67 & 0.33 \\
\hline 2005 & Wheat & 55 & 6,800 & $y=4.58 x^{1.78}$ & 0.49 & 0.73 & 0.27 \\
\hline 2005 & Barley & 43 & 3,500 & $y=5.73 x^{1.48}$ & 0.53 & 0.78 & 0.49 \\
\hline 2006 & Wheat & 48 & 10,000 & $y=5.43 x^{2.62}$ & 0.60 & 0.52 & 0.43 \\
\hline 2006 & Barley & 37 & 5,000 & $y=3.55 x^{1.55}$ & 0.14 & 0.64 & 0.08 \\
\hline
\end{tabular}

\subsection{Broad-scale annual yield prediction evaluation}

The broad-scale annual yield predictions all compare favourably with the reported yields for PIRSA regions (Figure 3). In general, the points in this scatter graph all fall close to the one-to-one line for predicted versus reported yield for wheat and barley, with 2004 and 2006 having the greatest dispersion around the line. Examining the data by year, the lowest yields were predicted and reported in 2006, the highest in 2005, and 
moderate yields were predicted and reported in 2004. Calculated $E$ values for 2004 and 2006 were moderate at around 0.35 while for $2005, E$ was around 0.65 .

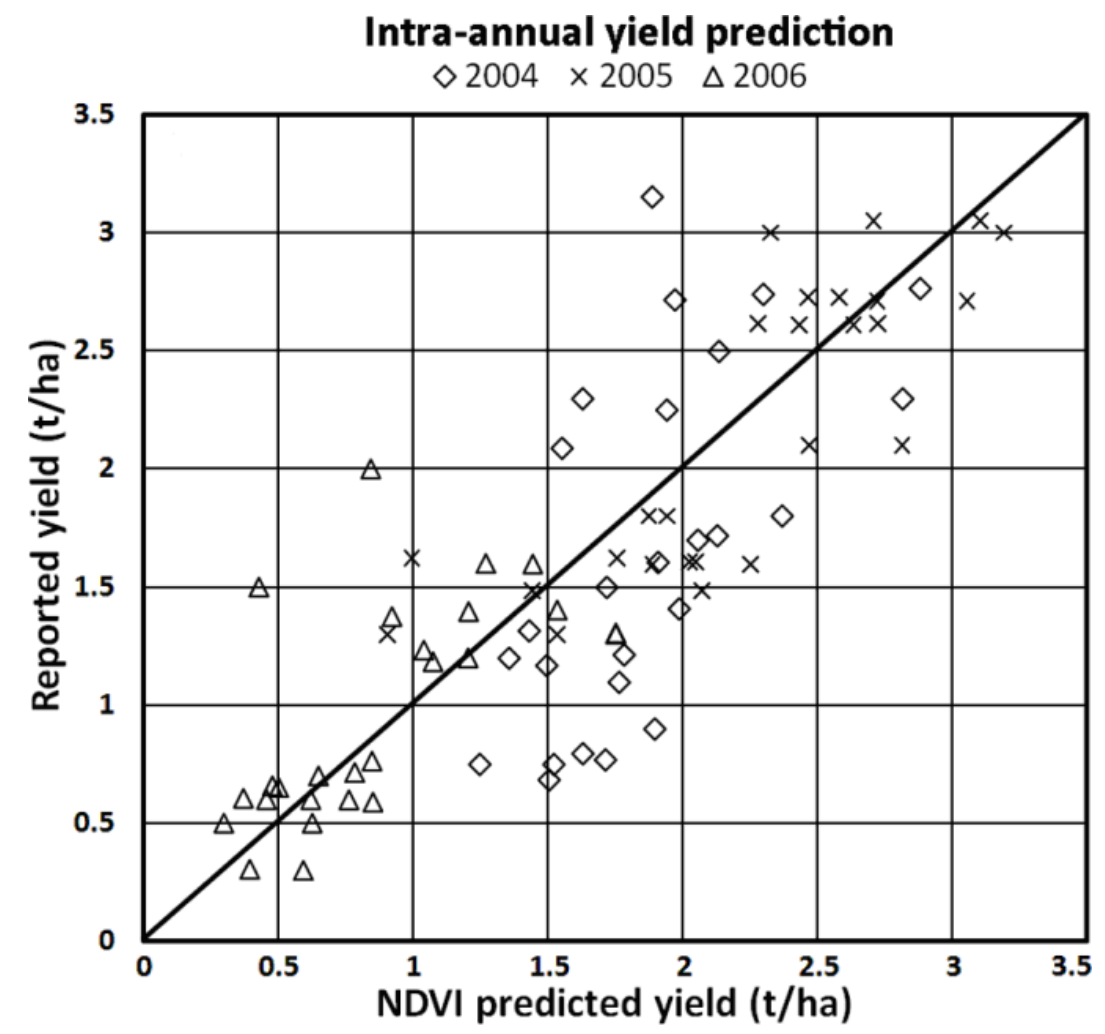

Figure 3. Scatter plot of regional yields predicted from yield-NDVI models versus yields reported to PIRSA for 2004, 2005 and 2006.

\section{DISCUSSION AND CONCLUSION}

The results presented in this paper suggest that it is possible to derive fairly robust fine scale wheat and barley models from yield data taken from a range of locations. The evaluation results suggest that these fine scale models can provide moderate extrapolation accuracy over space at a high resolution. These results are comparable to those found for models derived from yield data within specific geographic areas (Lyle et al., 2013). We chose simple logarithmic models to quantify the relationships between yield and NDVI. These models will under predict yield predictions at lower NDVI values (since the power curve is forced through the origin) and over predict yield at higher NDVI values. The extrapolation of these models across space can potentially skew yield values across the spectrum of NDVI values. There may also be some potential benefits to model fit if the datasets were transformed and non-linear modelling was used. Given our choice of models and their potential limitations, our analysis showed that that these yield-NDVI relationships can be spatially generalised outside the model training area to fairly accurately predict regional yields for the year in which the model was derived. This demonstrates that the models can be utilized for fine and coarse scale yield extrapolation over space. These results suggest that an extensive spatial distribution of yield data over different geographic regions is not needed to produce accurate yield estimates across these regions but that the variability contained in a very small spatial sample is generalizable for broad extents.

The very high correspondence of the broad scale predictions given the large variability in the yield regression models may be initially surprising given a low $\mathrm{R}^{2}$ in some of the yield models. Predictability of yield at the precision agriculture scale is substantially lower than the predictability of yield at a regional scale. This supports that NDVI well reflect differences in yield in a way that a geographically small spatial precision agriculture data sample can be used to accurately predict yield at the broad scale. These results imply that the general trend in the yield-NDVI relationship is unbiased irrespective of a very noisy point-based relationship between yield and NDVI. This noise can be explained by numerous sources of variation in yield measurement acquired in December/January (i.e. Lyle 2010) and differences in crop development and finishing after imagery acquisition in September, which may increase error in the yield-NDVI relationship. 
If it can be assumed that the regression error is due to the variability in yield, the results may mean that NDVI is a less noisy indicator of site conditions than yield. Hence pattern of NDVI may be of similar or even higher management relevance than yield pattern because management decisions rely on average site potential. This alone supports the need for future work on spatio-temporal variability of the yield-NDVI relationships.

The poor performance of the crop type classification can be explained by the lack of spectral difference between wheat and barley which would be apparent when our imagery was acquired. However, the inability to distinguish wheat fields from barley fields, and consequently the inability to apply the appropriate yieldNDVI relationship to each field did not prevent us from accurately predicting broad-scale annual regional yield, or broad-scale inter-annual regional yield. This may suggest that the yield-NDVI relationship for wheat is very similar to that for barley. This will be looked at in the future and if true then it may be unnecessary to attempt to distinguish between the two crop types in any future work.

\section{ACKNOWLEDGEMENTS}

We thank the NRM Research Alliance for funding this project and SPAA for access to their datasets.

\section{REFERENCES}

Blackmore, B. S. (2003). The role of yield maps in precision farming. National Soil Resources Institute. PhD Thesis. Cranfield University, Great Britain. 170pp.

Chandler, G., Markham, B. L. and Helder, D. L. (2009). Summary of current radiometric calibration coefficients for Landsat MSS, TM, ETM+, and EO-1 ALI sensors. Remote Sensing of Environment 113: 893-903.

Lyle G (2010) "I can't be green if I'm in the red": Combining precision agriculture and remote sensing technologies for sub field and regional decision making. School of Earth and Environmental Sciences. PhD Thesis. The University of Adelaide. Australia. 294pp.

Lyle, G., and Ostendorf, B. (2011) A high resolution broad scale spatial indicator of grain growing profitability for natural resource planning. Ecological Indicators, 11 (1), 209-218.

Lyle, G., Lewis, M., and Ostendorf, B. (2013) Testing the Temporal Ability of Landsat Imagery and Precision Agriculture Technology to Provide High Resolution Historical Estimates of Wheat Yield at the Farm Scale. Remote Sensing, 5 (4), 1549-1567.

Minasny, B., McBratney, A. B. and Whelan, B. M. (2005). VESPER version1.62 - Variogram Estimation and Spatial Prediction plus ERror. Australian Centre for Precision Agriculture. http://www.usyd.edu.au/su/agric/acpa/vesper/download.html

Nash, J. E. and Sutcliffe, J. V. (1970). River flow forecasting through conceptual models. Part 1 - A discussion of principles Journal of Hydrology 10: 282-290.

PIRSA (2009) Crop and Pasture Reports. Retrieved 19 August 2013. http://www.pir.sa.gov.au/grains/cpr.

Schut, A. G. T., Stephens, D. J., Stovold, R. G. H., Adams, M. and Craig, R. L. (2009). Improved wheat yield and production forecasting with a moisture stress index, AVHRR and MODIS data. Crop and Pasture Science 60(1): 60-70.

Taylor, J. A., McBratney, A. B. and Whelan, B. M. (2007). Establishing Management Classes for Broadacre Agricultural Production. Agronomy Journal 99: 1366-1376. 